\title{
Position Verification of the RADPOS 4-D In-Vivo Dosimetry System
}

\author{
Ryosuke Kohno1*, Hidenori Yamaguchi'2, Kana Motegi', Fumiya Tanaka1, \\ Tsunemichi Akita1, Yuki Nagata², Kenji Hotta1, Tomoko Miyagishi' ${ }^{1}$, Shie Nishioka1, \\ Takeshi Dohmae', Tetsuo Akimoto ${ }^{1}$ \\ ${ }^{1}$ Particle Therapy Division, National Cancer Center Hospital East, Kashiwa, Japan \\ ${ }^{2}$ Graduate School of Pure and Applied Sciences, University of Tsukuba, Tsukuba, Japan \\ Email: *rkohno@east.ncc.go.jp
}

Received 11 September 2015; accepted 8 November 2015; published 11 November 2015

Copyright @ 2015 by authors and Scientific Research Publishing Inc.

This work is licensed under the Creative Commons Attribution International License (CC BY). http://creativecommons.org/licenses/by/4.0/

c) (†) Open Access

\section{Abstract}

The accuracy of the position measurements obtained by the radiation positioning system (RADPOS) was evaluated under static and dynamic conditions. In the static verifications, the RADPOS was fixed to the treatment couch in a photon treatment room and a proton treatment room, and was translocated with the treatment couch in $\mathrm{x}, \mathrm{y}$ and $\mathrm{z}$ directions. Because the presence of magnetic and/or electrically conductive materials can cause a systematic shift in the measured position by distorting the RADPOS transmitted field, the effect of metals on the performance of the positioning system was also investigated. Dynamic verification was performed using the couch drive and a dynamic anthropomorphic thorax phantom. We thus confirmed the utility of RADPOS as a position sensor to perform in vivo dosimetry.

\section{Keywords}

RADPOS, MOSFET, In-Vivo Dosimetry, Position Sensor, Photon Therapy, Proton Beam Therapy

\section{Introduction}

The goal of radiation therapy is to deliver a highly conformal dose to the tumor, while at the same time sparing the surrounding normal tissues. Accurate dose delivery is important, and is assured by measuring the radiation delivered to the patient. Therefore, in vivo dosimetry serves as the ultimate dose verification for patient quality assurance.

To carry out in-vivo dosimetry, the detector must be very small and easy to localize. The metal oxide semi-

\footnotetext{
${ }^{*}$ Corresponding author.
}

How to cite this paper: Kohno, R., et al. (2015) Position Verification of the RADPOS 4-D In-Vivo Dosimetry System. International Journal of Medical Physics, Clinical Engineering and Radiation Oncology, 4, 318-325. 
conductor field-effect transistor (MOSFET) dosimeter [1] has been widely studied for use as a dosimeter for patient dose verification [2]-[4]. However, Kohno et al. reported that large measurement errors are unavoidable when performing in vivo dosimetry, because it is difficult to measure accurate point doses [5]. To perform meaningful in vivo dosimetry, we have to monitor accurately the position of the MOSFET detector.

The radiation positioning system (RADPOS) consists of a MOSFET dosimeter coupled with an electromagnetic positioning device (Best Medical Canada, Ottawa, ON). The RADPOS can be used for simultaneous position and dose measurement [6]-[8]. The accuracy of the RADPOS position measurement is $1.4 \mathrm{~mm}$ root mean square (rms), within the performance motion range defined by the manufacturer: $x=200$ to $360 \mathrm{~mm}$ from the transmitter center, $y= \pm 200 \mathrm{~mm}$ from the transmitter center, and $z= \pm 200 \mathrm{~mm}$ from the transmitter center [9].

One drawback of the RADPOS is that metal objects near the transmitter or the sensors will adversely affect the positioning accuracy. The presence of magnetic and/or electrically conductive materials can cause a systematic shift in the measured position by distorting the RADPOS transmitted field. The magnitude of the distortion caused by a material can depend on the material's ferromagnetism, electrical conductivity, and physical shape. Therefore, the presence of metal objects or structures in the vicinity of the transmitter and sensors should be avoided. Nonetheless, RADPOS shows promise as a means to improve the accuracy of in vivo dosimetry.

Our ultimate goal is to achieve in-vivo proton dosimetry. However, there are no reports to date about the use of the RADPOS for proton beam therapy (PBT). Since the equipment for PBT contains metal parts in the patient collimator and nozzle apparatus, there is the concern that this may negatively affect the accuracy of the position measurements obtained via RADPOS. In this paper, we carried out static and dynamic verification of the accuracy of the position measurements obtained via RADPOS. Evaluations of RADPOS in an environment relevant to PBT were also performed.

\section{Materials and Methods}

\subsection{RADPOS System}

A schematic of the RADPOS is shown in Figure 1. The RADPOS system consists of a MOSFET radiation dosimeter and an electromagnetic positioning device. The MOSFET has an active area of $0.2 \times 0.2 \mathrm{~mm}^{2}$. The dose measurement is based on the change in threshold voltage $\Delta V_{\text {th }}$ before and after irradiation. The electromagnetic positioning sensor is a small cylinder $8 \mathrm{~mm}$ in length and $1.3 \mathrm{~mm}$ in diameter. To avoid radiation attenuation and disturbance, the positioning sensor is separated by $8 \mathrm{~mm}$ from the MOSFET dosimeter.

The RADPOS probe is connected to a mobile MOSFET reader to record the threshold voltage of the dosimeter. The probe is also connected to a 3D Guidance preamplifier and 3D Guidance tracker. The 3D Guidance DC magnetic field transmitter, which is connected to the 3D Guidance tracker, generates a pulsed 3D magnetic field with well-defined characteristics. The response of the sensor to this magnetic field is monitored by the position tracker and analyzed to determine the $x, y, z$ coordinates as well as the azimuth, elevation, and roll rotations of the RADPOS probe.

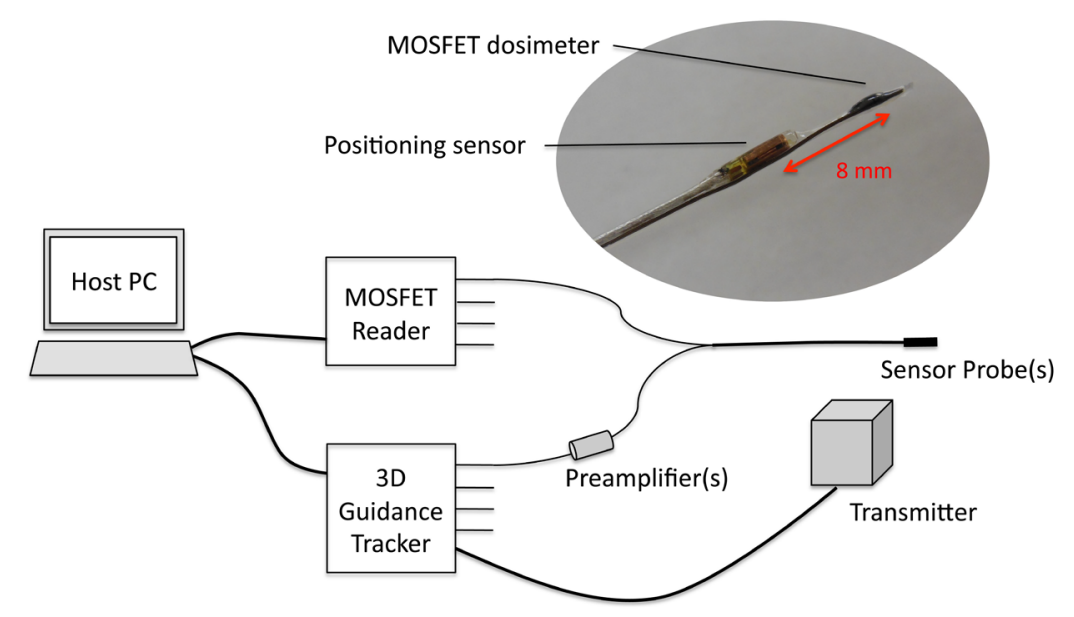

Figure 1. Schematic of RADPOS dosimetry system. 
The MOSFET reader and 3D Guidance tracker are connected to a host computer using either a direct connection or wireless technology. Special software has been developed which allows the user to record the $\Delta V_{t h}$ of the MOSFET and the spatial coordinates of the position sensor either manually or automatically at user-defined time intervals. The RADPOS software determines the axes of the coordinates by the orientation of the transmitter, as shown in Figure 2. The transmitter was positioned so that the $x, y$, and $z$ axes corresponded to the head-foot, left-right, and anterior-posterior direction, respectively.

\subsection{Static Position Verification}

The accuracy of the RADPOS position measurements was evaluated in a photon treatment room and a proton treatment room. The RADPOS was fixed to the treatment couch, and translocated with the treatment couch in the $\mathrm{x}, \mathrm{y}$ and $\mathrm{z}$ directions. The origin was defined as the point $200 \mathrm{~mm}$ from the transmitter center along the $x$ direction. The quantity of movement of the RADPOS was compared to the quantity of drive of the treatment couch.

The 550 TxT Treatment Table (Siemens AG, Inc. Erlangen, Germany) was used in the photon treatment room, and this movement accuracy specification was $\pm 0.5 \mathrm{~mm}$. The $\mathrm{x}$-axis scan ranged from -100 to $400 \mathrm{~mm}$, the $\mathrm{y}$-axis scan ranged from -250 to $250 \mathrm{~mm}$, and the scan along the z-axis ranged from -400 to $200 \mathrm{~mm}$. In this study, measurements were carried out all three times, error bars are calculated as one standard deviation.

A treatment couch (Sumitomo Heavy Industries, Ltd., Tokyo, Japan) in the proton treatment room was used. Accuracy of the couch had previously been confirmed to be less than $1.0 \mathrm{~mm}$ by a monthly QA. The proton gantry will be fitted with nozzle. The functions of the nozzle include the beam shaping, the beam monitoring and dosimetry. The beam line employed a single-ring wobbling spreading method. The snout of the nozzle is telescopic to allow the bolus and the patient collimator to be placed as close as possible to the patient, minimizing deterioration of the penumbra. The snout position was $-400 \mathrm{~mm}$ along the $\mathrm{z}$-axis from the couch. The $\mathrm{x}$-axis scan ranged from -100 to $100 \mathrm{~mm}$, the $\mathrm{y}$-axis scan ranged from -400 to $400 \mathrm{~mm}$, and the scan along the z-axis ranged from -360 to $160 \mathrm{~mm}$. In addition, we also investigated the effect of the beam-wobbling magnets on the RADPOS signal.

To study the effect of metals on the RADPOS signal, we used square samples of aluminum, nonmagnetic stainless steel and brass, each $25 \times 25 \mathrm{~cm}^{2}$ and $6 \mathrm{~mm}$ in thickness. The choice of samples studied for interference was determined by the materials encountered in the proton treatment room. The initial RADPOS position in Figure 2 was recorded and zeroed. Each sample was positioned along the $x$-axis at distances ranging from 20 to $300 \mathrm{~mm}$ away from the RADPOS. At each position, the positional error was evaluated.

A further consideration in the clinical application of this system is the patient collimator, which is made of brass. To estimate its maximum effect, we used a patient collimator with a relatively small opening size of $2 \mathrm{~cm}$ in diameter. The RADPOS was positioned at the isocenter on the treatment couch, and we recorded the position of the RADPOS as the standard position. The RADPOS position was read simultaneously with that of the snout

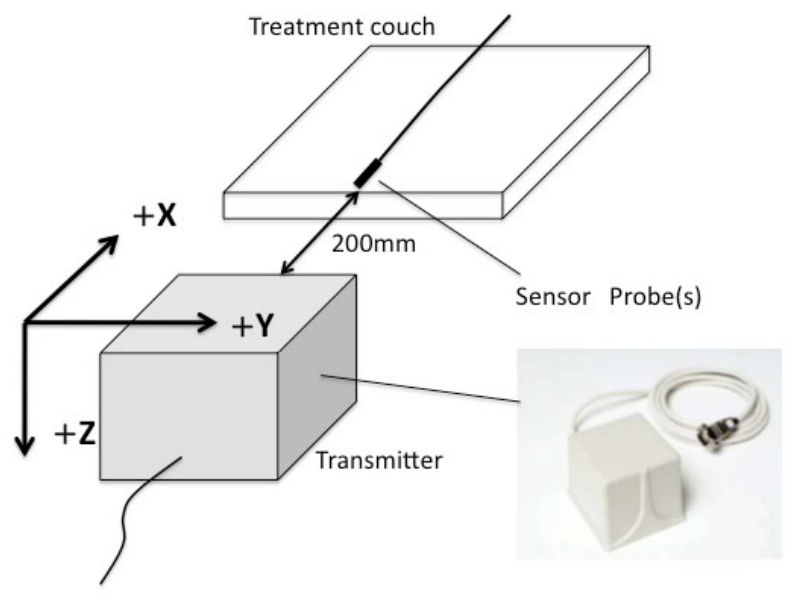

Figure 2. The RADPOS transmitter, sensor probe and coordinate system. 
with the patient collimator, which was moved in $100 \mathrm{~mm}$ increments over a range of 100 to $400 \mathrm{~mm}$ away from the RADPOS along the z-axis.

\subsection{Dynamic Position Verification}

A useful characteristic of the RADPOS is that it can detect position in real time. We fixed the RADPOS to a robotic couch (Forte, Machesney Park, IL) whose accuracy had previously been confirmed to be less than $0.5 \mathrm{~mm}$ by a monthly QA, and verified the accuracy of its dynamic position measurement. The RADPOS was translocated with the treatment couch in the $\mathrm{z}$ direction at 10,20 and $30 \mathrm{~mm} / \mathrm{s}$. The RADPOS can record a measured position every $0.1 \mathrm{~s}$. The drive speed of the couch was calculated from each measured position, and compared with the setting speed of the couch.

2 RADPOS probes (A, B) were fixed to a surface of a dynamic anthropomorphic thorax phantom (Figure 3). A lung balloon inside a chest wall in the dynamic anthropomorphic thorax phantom was inflated and deflated by a computer-controlled air pump. A breathing signal is sent from the laptop computer. This dynamic anthropomorphic thorax phantom was made in house, and we have confirmed respiratory motion reproducibility of within $0.3 \mathrm{~mm}$. This phantom was scanned by using the Aquilion ONE computed tomography (CT) scanner (Toshiba Medical Systems, Otawara, Tochigi, Japan). The 4DCT images were retrospectively reconstructed with 10 equally-spaced phases, with $0 \%$ phase defined as end-inhalation. The coordinate of the probe was calculated from each obtained image, relative to a standard position which we defined as the probe position in $0 \%$ phase. The probe position in each phase relative to the probe position in $0 \%$ phase was calculated, and compared with the position measured by the RADPOS. This series of measurements was carried out three times.

\section{Results and Discussions}

\subsection{Static Position Verification}

Figure 4 shows the RADPOS position error along the $\mathrm{x}, \mathrm{y}$ and $\mathrm{z}$-axes in the photon room. In the $\mathrm{x}$ direction, the average absolute deviation within the range $0-160 \mathrm{~mm}$ was $0.4 \pm 0.3 \mathrm{~mm}$ with a maximum deviation of $0.9 \mathrm{~mm}$. In y direction, the average absolute deviation within the range $-200-200 \mathrm{~mm}$ was $0.4 \pm 0.4 \mathrm{~mm}$ with a maximum deviation of $1.1 \mathrm{~mm}$. In the $\mathrm{z}$ direction, the average absolute deviation within the range $-200-200 \mathrm{~mm}$ was $0.3 \pm 0.2 \mathrm{~mm}$ with a maximum deviation of $0.8 \mathrm{~mm}$. These results were within the manufacturer's specifications of $1.4 \mathrm{~mm}$ rms accuracy, and better position accuracy than those of Cherpak et al. [6]. Since the position accuracy depends on a measurement environment, it should be verified in advance in an environment in which the RADPOS is used. Incidentally, the RADPOS error was more than $1.5 \mathrm{~mm}$ when operating outside of the range of motion defined by the manufacturer.

The RADPOS position error in the proton room is shown in Figure 5. In the $x$ direction, the average absolute deviation within the range $0-100 \mathrm{~mm}$ was $0.9 \pm 0.8 \mathrm{~mm}$ with a maximum deviation of $2.1 \mathrm{~mm}$. In the y direction, the average absolute deviation within the range $-200-200 \mathrm{~mm}$ was $0.4 \pm 0.4 \mathrm{~mm}$ with a maximum deviation of $1.5 \mathrm{~mm}$. In the $\mathrm{z}$ direction, the average absolute deviation within the range $-200-200 \mathrm{~mm}$ was $0.6 \pm 0.5$ $\mathrm{mm}$ with a maximum deviation of $1.6 \mathrm{~mm}$. Although these results were close to the manufacturer's specifica-

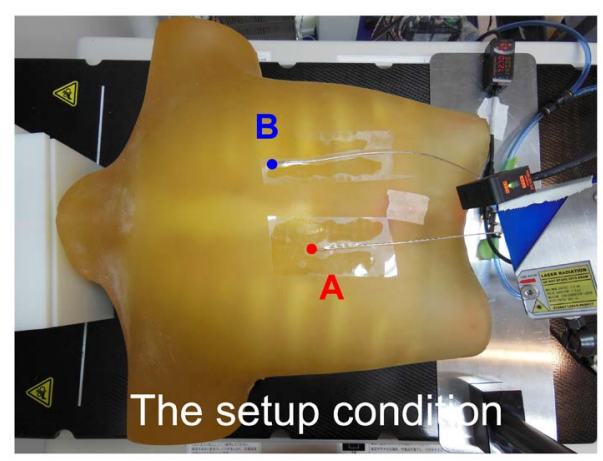

Figure 3. Placement of the RADPOS probes ((A), (B)) on the surface of a dynamic anthropomorphic thorax phantom. 


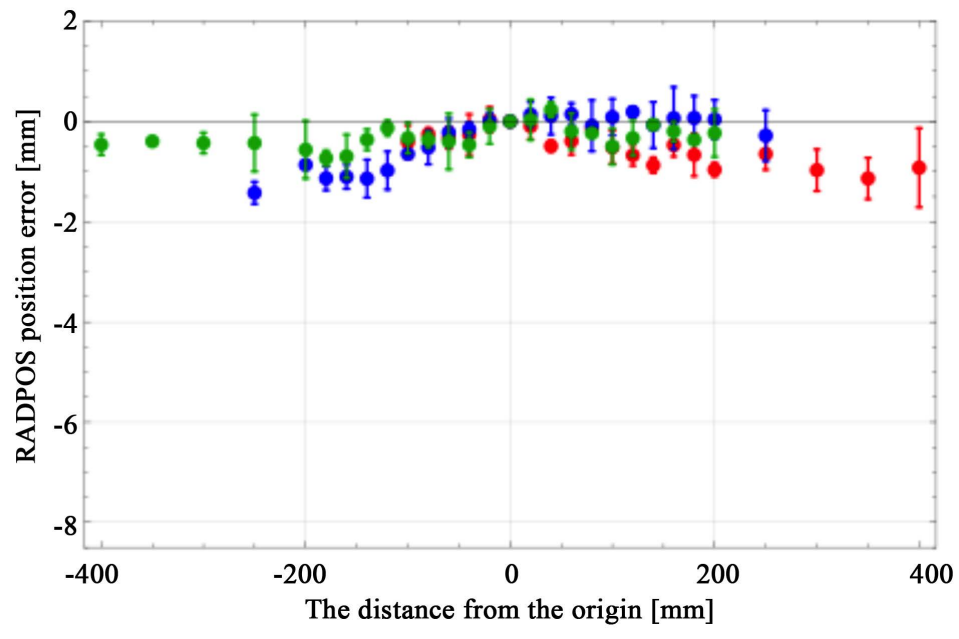

Figure 4. The RADPOS position error along the $x$ (red), $y$ (blue) and $z$ (green)axes in the photon room.

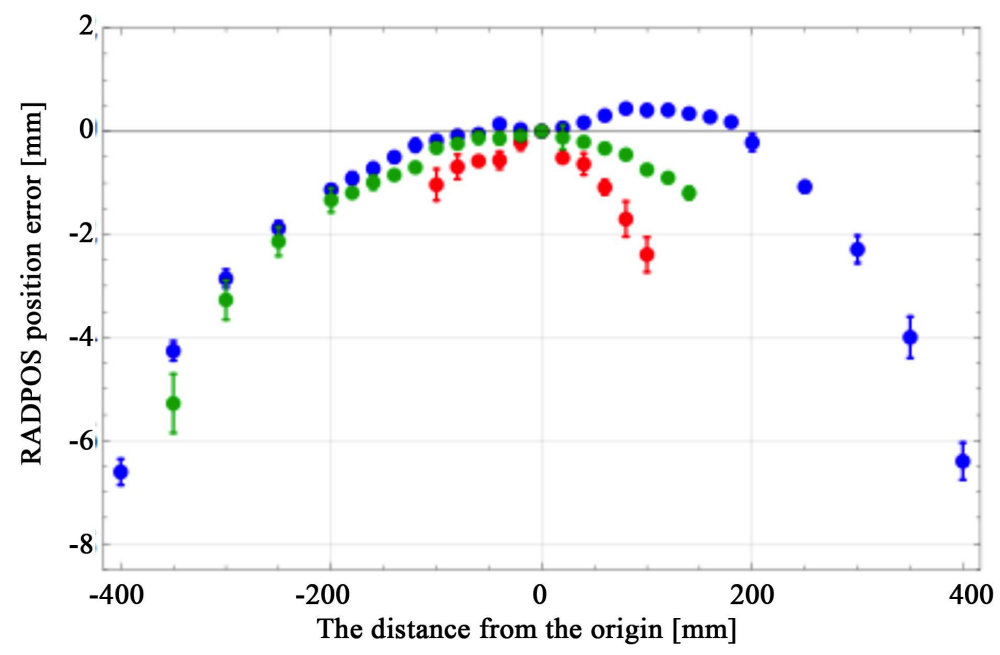

Figure 5. The RADPOS position error along the $x$ (red), $y$ (blue) and $z$ (green)axes in the proton room.

tions, they were generally worse than the results obtained in the photon room. Then, we did not observe an effect of the beam-wobbling magnets within $\pm 0.1 \mathrm{~mm}$. This is likely because the distance between the magnets and the isocenter is relatively large, $2.6 \mathrm{~m}$ for one magnet and $2.3 \mathrm{~m}$ for the other magnet.

Figure 6 shows the effect of the interfering materials on the RADPOS signal. Aluminum and brass caused large distortions, while non-magnetic stainless steel caused no distortion. The distortions were reduced to a negligible amount when the separation between the detector and sample was at least $200 \mathrm{~mm}$. This result was the same as the paper of Cherpak et al. [6].

The relationship between the snout position and the RADPOS position error is shown in Figure 7. The error along all axes was largest when the snout position was $100 \mathrm{~mm}$ from the RADPOS, and decreased as the snout was moved further away. In particular, the accuracy of the z-axis deteriorated considerably. From additional research on the impact of the snout due to the presence or absence of the patient collimator for the RADPOS position accuracy, it was obvious that the influence of the snout itself with nozzle apparatus occupies about $80 \%$ of the RADPOS position error. Since the equipment for PBT contains many metal parts in the snout, the position accuracy of the RADPOS should be verified in advance in an environment in which the RADPOS is used.

Finally, when the snout position was more than $400 \mathrm{~mm}$ away from the RADPOS, the position error was less 


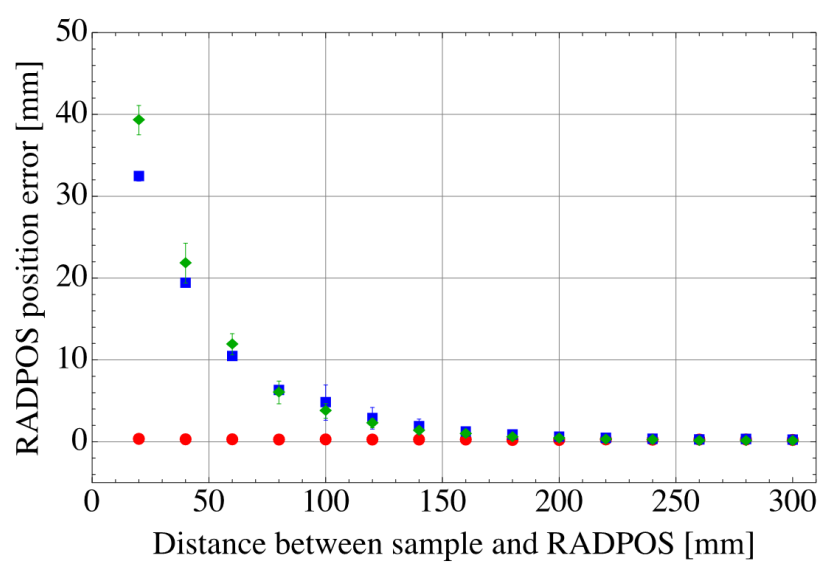

Figure 6. The effect of the interfering materials (aluminum: green, brass: blue, steel: red) on the RADPOS signal.

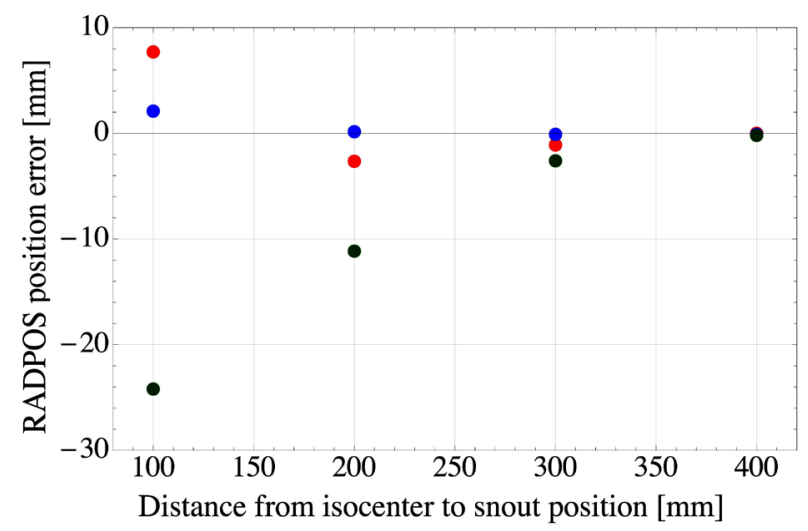

Figure 7. Relationship between the snout position and the RADPOS position error along the $\mathrm{x}$ (red), $\mathrm{y}$ (blue) and $\mathrm{z}$ (green)-axes.

than $1 \mathrm{~mm}$. Special attention must therefore be paid when using the RADPOS in cases where the snout position is close to the patient, such as during treatment of the head and neck. Thus, there are some problems of the snout position and nozzle apparatus in use of the RADPOS. However, in separating the distance with them like prostate irradiation condition, we think that better accuracy of in-vivo proton dosimetry can be obtained, since the RADPOS positions can be measured in real-time during irradiation.

\subsection{Dynamic Position Verification}

RADPOS measured drive speeds of the couch as 10, 19.9 and $29.9 \mathrm{~mm} / \mathrm{sec}$, which agreed with the actual drive speed to within $0.1 \mathrm{~mm} / \mathrm{sec}$. It is clear RADPOS is a viable method to perform dynamic position measurement.

Figure 8 shows comparisons of the positions measured by the RADPOS and the RADPOS positions obtained by the 4DCT on the dynamic anthropomorphic thorax phantom. The RADPOS precisely detected the movement of the dynamic anthropomorphic thorax phantom. The maximum positions measured by the RADPOS at measurement points A and B were $6.3 \pm 0.1 \mathrm{~mm}$ and $5.5 \pm 0.1 \mathrm{~mm}$, respectively, and those obtained by the 4DCT were $6.2 \pm 0.1 \mathrm{~mm}$ and $5.4 \pm 0.1 \mathrm{~mm}$, respectively. Cherpak et al. reported difference between the set amplitude and the measured one for a sinusoidal motion of the Quasar phantom was $0.2 \pm 0.3 \mathrm{~mm}$ [6], and we got almost the same position accuracy with them. Thus, the RADPOS can monitor body movement of a patient to within $0.1 \mathrm{~mm}$ during irradiation.

Modern radiation therapy technology is in principle capable of delivering highly conformal radiation beams focused on the tumor. In practice, the patient body is not static. Therefore, some movement of the tumor due to breathing and setup errors exists between interfraction motions and intrafraction motions [10]-[12]. As results of 


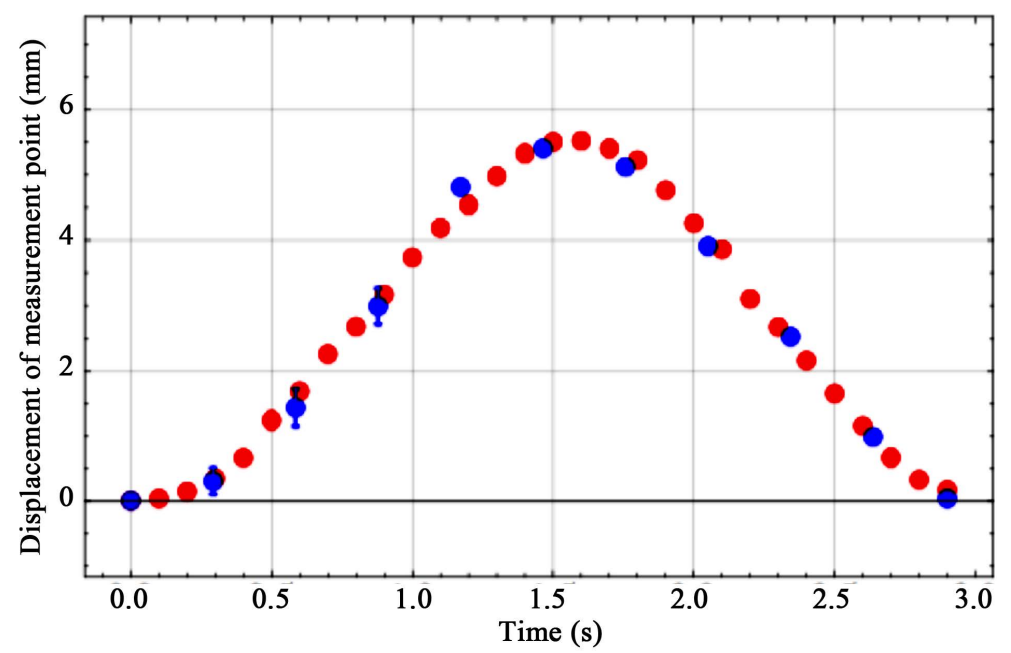

(A)

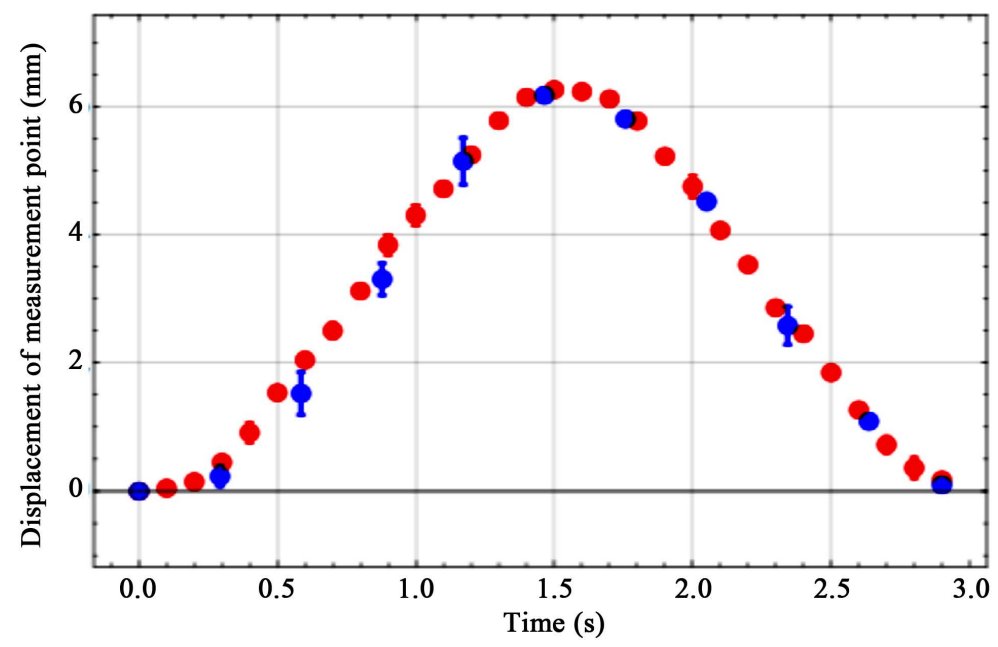

(B)

Figure 8. Comparison of the positions measured by the RADPOS (red) versus the RADPOS positions obtained by the 4DCT (blue) on the dynamic anthropomorphic thorax phantom.

the dynamic position verification show, using the RADPOS to continuously monitor patient position during irradiation can provide valuable information without radiation exposure.

\section{Conclusion}

The accuracy of RADPOS position measurements was evaluated by static and dynamic position verifications. The RADPOS position error was within $1.0 \mathrm{~mm}$ along the $\mathrm{x}, \mathrm{y}$ and $\mathrm{z}$-axes within the performance motion range defined by the manufacturer, providing clear evidence that the RADPOS can be useful as a position sensor. However, during application of PBT, interfering materials such as the snout in the beam-delivery system can distort the RADPOS transmitted field. Although special attention is necessary when using the RADPOS in PBT, RADPOS positions can be measured in real-time during irradiation. We try to perform better dose verification with the RADPOS for PBT in the near future.

\section{Acknowledgements}

The authors would also like to thank Masaki Shinoda, Hiroyuki Suzuki, Tetsuro Kawaguchi, Satoshi Kai, Atsu- 
shi Sakamoto, Hideki Watanabe and Kazuo Sugimori,, SHI Accelerator Service Ltd., for experimental support. This work was supported in part by a Grant-in-Aid for Young Scientists (A) (No. 23689058) from the Japan Society for Promotion of Science (JSPS). This study was also supported in part by the National Cancer Center Research and Development Fund (25-A-10) and Health Science Research Grants from the Ministry of Health and Welfare (26270701). This research is also supported by the "Development of Medical Devices and Systems for Advanced Medical Services" from Japan Agency for Medical Research and development, AMED (15ck0106033h0002).

\section{References}

[1] Soubra, M., Cygler, J. and Mackay, G. (1994) Evaluation of a Dual Metal Oxide-Silicon Semiconductor Field Effect Transistor Detector as Radiation Dosimeter. Medical Physics, 21, 567-572. http://dx.doi.org/10.1118/1.597314

[2] Marcie, S., Charpiot, E., Bensadoun, R.J., Ciais, G., Herault, J., Costa, A. and Gerard, J.P. (2005) In Vivo Measurements with MOSFET Detectors in Oropharynx and Nasopharynx Intensity-Modulated Radiation Therapy. International Journal of Radiation Oncology Biology Physics, 61, 1603-1606. http://dx.doi.org/10.1016/j.ijrobp.2004.12.034

[3] Beyer, G.P., Scarantino, C.W., Prestidge, B.R., Sadeghi, A.G., Anscher, M.S., Miften, M., Carrea, T.B., Sims, M. and Black, R.D. (2007) Technical Evaluation of Radiation Dose Delivered in Prostate Cancer Patients as Measured by an Implantable MOSFET Dosimeter. International Journal of Radiation Oncology Biology Physics, 69, 925-935. http://dx.doi.org/10.1016/j.ijrobp.2007.06.065

[4] Bloemen-van Gurp, E.J., Mijnheer, B.J., Verschueren, T.A. and Lambin, P. (2007) Total Body Irradiation, toward optimal Individual Delivery: Dose Evaluation with Metal Oxide Field Effect Transistors, Thermoluminescence Detectors and a Treatment Planning System. International Journal of Radiation Oncology Biology Physics, 69, 1297-304. http://dx.doi.org/10.1016/j.ijrobp.2007.07.2334

[5] Kohno, R., Hotta, K., Matsubara, K., Nishioka, S., Matsuura, T. and Kawashima, M. (2012) In Vivo Proton Dosimetry Using a MOSFET Detector in an Anthropomorphic Phantom with Tissue Inhomogeneity. Journal of Applied Clinical Medical Physics, 13, 159-167.

[6] Cherpak, A., Ding, W., Hallil, A. and Cygler, J.E. (2009) Ecvaluation of a novel 4D in Vivo Dosimetry System. Medical Physics, 36, 1672-1679. http://dx.doi.org/10.1118/1.3100264

[7] Cherpak, A., Serban, M., Seuntjens, J. and Cygler, J.E. (2011) 4D Dose-Position Verification in Radiation Therapy Using the RADPOS System in a Deformable Lung Phantom. Medical Physics, 38, 179-187. http://dx.doi.org/10.1118/1.3515461

[8] Cherpak, A., Cygler, J.E., Andrusyk, S., Pantarotto, J., Macrae, R. and Perry, G. (2012) Clinical Use of a Novel in Vivo 4D Monitoring System for Simultaneous Patient Motion and Dose Measurements. Radiotherapy and Oncology, 102, 290-296. http://dx.doi.org/10.1016/j.radonc.2011.08.021

[9] Best Medical Canada. Operator’s Manual for the RadPos 4-D in-Vivo Dosimetry System. Health Canada: Medical Device Licence No. 84809.

[10] Langen, K.M. and Jones, D.T.L. (2001) Organ Motion and Its Management. International Journal of Radiation Oncology Biology Physics, 50, 265-278. http://dx.doi.org/10.1016/S0360-3016(01)01453-5

[11] Jones, D. (1993) ICRU Report 50, Prescribing, Recording, and Reporting Photon Beam Therapy. International Commission on Radiation Units and Measurements, Bethesda, Maryland.

[12] Webb, S. (2006) Motion Effects in Intensity Modulated Radiation Therapy: A Review. Physics in Medicine and Biology, 51, R403-R425. http://dx.doi.org/10.1088/0031-9155/51/13/r23 